\title{
Strategic Innovation for Business Performance: The Art and Science of Transformation
}

\author{
Harold Schroeder
}

\author{
"If you want something new, you have to stop doing") \\ something old.
}

Peter F. Drucker (1909-2005)

Author and management consultant

\begin{abstract}
Despite the well-documented association between innovation and business performance, many organizations struggle in their attempts to become successful innovators. This article discusses a recommended "art and science of transformation" approach to help companies improve their innovation performance through effective organizational change. The approach is focused on four key factors: culture, collaboration, strategy, and systems. Examples are drawn from a review of previous research to demonstrate successful innovation practice using similar approaches, and examples of less successful practice are included to highlight ways in which an "art and science" approach can help overcome the difficulties often faced. The article concludes with some practical, step-by-step guidance based on the art and science of transformation framework.
\end{abstract}

\section{Introduction}

Numerous studies have highlighted the importance of innovation as a critical success factor in business performance (e.g., Baker and Sinkula, 2002: tinyurl.com/ qb9ht7l; Damanpour et al., 1989: tinyurl.com/plm9gp2; Hult et al., 2004: tinyurl.com/nyns9qa; Jiménez-Jimenez et al., 2008: tinyurl.com/khxlmjl; Roberts, 1999: tinyurl.com/ksapsre). Leading global corporation such as Apple Inc., 3G, and Proctor \& Gamble largely owe their outstanding business success to a sustained record of successful innovation. Yet, despite the well-documented association between innovation and business performance, many companies struggle in their attempts to become successful innovators.

The available evidence shows that the companies that are most successful at innovation approach it in a holistic and systematic way, developing an innovation strategy that is fully integrated with their business mission and goals, and aligning their organizational culture and organizational systems with the strategy. Relatively few organizations take this approach; however, if innovation occurs at all, it is more often in an ad hoc fashion that has little connection to core business goals (De
Souza et al., 2009: tinyurl.com/mzxbdj5; Jaruzelski et al., 2011: tinyurl.com/lysol6j). In the PricewaterhouseCoopers global survey of CEOs (2011; tinyurl.com/4dboztd), fewer than $10 \%$ of respondents described their organization as an "active innovator".

Developing a business environment that supports and promotes innovation often requires extensive changes in organizational culture and systems, which can be difficult to achieve, not to mention disruptive, costly, and time-consuming. Though the potential long-term benefits are considerable, firms are often focused on shortterm gains and cost reductions and are unwilling to invest time and resources into organizational transformation efforts. The high risks of failure associated with major organizational change projects may also be a deterrent.

This article discusses an "art and science" approach to help companies improve their innovation performance through effective organizational transformation. First, the article describes the overall approach, and then it discusses of each of its four key factors: culture, collaboration, strategy, and systems. Next, examples of less successful attempts to innovate are provided to illus- 


\section{Strategic Innovation for Business Performance: The Art and Science of Transformation} Harold Schroeder

trate the risks of not paying sufficient attention to these factors. Finally, the article concludes with recommendations for organizations wishing to improve their innovation performance using the art and science of transformation framework.

\section{The Art and Science of Transformation}

The approach described in this article is based on growing evidence that successful organizational change needs a combination of art and science, with science comprising specialist expertise and techniques and art comprising the more intangible and intuitive types of skills that are at least as important. In general, the art of transformation focuses mainly on the cultural and people-related aspects of change, and the science focuses on the strategic and systems-related aspects, but with considerable overlaps. The research evidence shows that organizational transformation projects often fail because of a lack of attention to the people-related aspects of change (Economist Intelligence Unit, 2009: tinyurl.com/lmwyevv; IBM Corporation, 2008: tinyurl .com/ob8nvym; Bisson et al., 2010: tinyurl.com/oqegoyf) rather than inadequate project planning and management.

Building on this art and science perspective, we can identify from previous research four main factors that are especially important in improving innovation performance: culture, collaboration, strategy, and systems. Loosely, two of these fall in the realm of art and two in the realm of science (Table 1), but they are closely interrelated. For example, a desired cultural change can be accomplished by disseminating new ideas and involving employees in the application of these ideas in their own areas of work, eventually resulting in changes in the underlying norms that direct day-to-day behaviour. However, the success of this effort also requires organizational systems - such as the rewards and recognition system, the performance management system, and leadership models - to be re-aligned with the desired new cultural norms. Attempts to persuade em-

Table 1. Key factors in the "art and science of transformation" approach to innovation

\begin{tabular}{cc}
\hline The Art & The Science \\
\hline Organizational Culture & Strategy \\
Collaboration & Systems \\
\hline
\end{tabular}

ployees to become more innovative are unlikely to succeed within a strongly hierarchical organizational structure that inhibits them from putting forward their own ideas, or if the performance evaluation system does not reward creativity.

An "art and science" approach to transformation is also required within each of these areas. For example, specialist human resources knowledge and skills are needed to redesign performance-management systems in ways that promote innovative behaviour, but this redesign also requires the intuitive ability to understand what will motivate different groups of employees and effectively communicate the changes to them. Cultural changes require the ability to influence the attitudes of employees towards the newly redesigned organizational systems and to encourage behavioural changes, but this cannot take place in an ad hoc manner; like any other transformation initiative, effective cultural change requires the application of systematic project planning and management techniques and the ability to monitor and report on progress. Art and science can also be conceptualized as "right brain" and "left brain" thinking, respectively, with the former being more intuitive, holistic, and subjective, and the latter more logical, analytical and objective - both are needed to gain full understanding of an issue and develop the most appropriate solution.

The following sections discuss the role of each of these factors in contributing to innovation performance, and explain how art and science are important in each area, highlighting also the inter-relationships between them.

\section{Organizational Culture}

Organizational culture consists of values, norms, and behaviours, which collectively define and comprise acceptable and "normal" ways of getting things done within an organization. Research has consistently shown organizational culture to be strongly associated with successful innovation. For example, a 2007 global survey of more than 700 public companies from $17 \mathrm{de}-$ veloped and developing economies revealed culture as the single most important driver of innovation, exceeding even $R \& D$ spending in its influence (Tellis et al., 2007; tinyurl.com/lgfxmlp). Similarly, Booz \& Company's Global Innovation 1000 study found organizational culture and strategic alignment to be the critical success factors in innovation (Jaruzelski et al., 2011; tinyurl.com/ lysol6j). In their research with companies in the San Francisco Bay Area, Jaruzelski, Merle, and Randolph (2012; tinyurl.com/m9065uo) found evidence of a "distinct 


\section{Strategic Innovation for Business Performance: The Art and Science of Transformation} Harold Schroeder

culture of innovation" that helps to align an organization's innovation approach with their business strategy.

Having a future-market orientation and a learning culture are the cultural attributes most strongly linked with the ability to innovate (Hult et al., 2004: tinyurl.com/nyns9qa; Hurley and Hult, 1998: tinyurl.com/ k358et9; Jiménez-Jimenez et al., 2008: tinyurl.com/khxlmjl; Nonaka and Takeuchi, 1995: tinyurl.com/qjjkltz). A market orientation requires that executives and other key employees have an astute "market sense" or understanding of evolving market needs and preferences. It also requires the ability to determine the right time to introduce a new product to the market - which may not necessarily be when it has been perfected in the laboratory. For example, Microsoft reportedly operate a practice of putting new products on the market before all the kinks have been ironed out, and subsequently improving them based on customer feedback. However, a science-based approach is also important to underpin and strengthen a firm's market orientation, including, for example, the use of market intelligence and analytics.

An organizational-learning orientation requires the presence of organizational norms and values that support experimentation and risk-taking. Tolerance of failure is an important aspect of a culture that supports organizational learning: from this perspective all experiments generate useful knowledge, even if they do not result in a concrete positive outcome. This attitude is reflected in the approach taken by Walmart, which views each of its stores as a mini-laboratory in which employees experiment with different pricing, product selection, and display options, and the most successful ideas are rolled out to the whole company (Leavy, 2005; tinyurl.com/oww5fdv).

Other cultural factors known to be associated with successful innovation include "a willingness to cannibalize"; risk tolerance; openness to ideas from external stakeholders; good collaboration; employee pride in the company's products and services; and respect for technical expertise (Jaruzelski et al., 2012: tinyurl.com/ m9065uo; Tellis et al., 2007; tinyurl.com/lgfxmlp).

Leaders and managers play a pivotal role in determining the culture of an organization by acting as role models or communicators of desirable norms and behaviour, and also in the ways they react to employee creativity. De Souza and colleagues (2009; tinyurl.com/ mzxbdj5) highlight the case of Whirlpool, in which the solid support of organizational leadership for the company's innovation strategy and infrastructure, including the allocation of seed funding for pilot projects and the communication of slogans promoting the importance of innovation to the organization, contributed significantly to the development of a highly innovative culture.

Organizational structures and systems are also especially important in supporting a learning culture. Examples include a structure that promotes team-working as well as collaboration between different areas of the organization; information sharing and knowledge transfer facilities or mechanisms; and performance-management systems and career paths that reward experimentation and knowledge generation (Nonaka and Takeuchi, 1995; tinyurl.com/qjjkltz). Efforts to encourage a more innovative culture can also be formalized in company rules and regulations: Google, for example, allows its employees to work on innovative ideas for $20 \%$ of their working time (Phillips, 2012; tinyurl.com/nykto5z).

\section{Collaboration}

The second major factor strongly associated with successful innovation is the ability to form relationships with internal and external stakeholders for the purpose of collaborating with or engaging them in the innovation process. Indeed, innovation performance has been shown to be correlated with the strength of a firm's network (Chetty and Stangl, 2010: tinyurl.com/kwng230; Mohannak, 2007: tinyurl.com/19uflzh; Vithessonthi, 2010: tinyurl.com/mkco7t6).

Collaboration takes place in the innovation process for two main reasons: i) to understand and incorporate the needs and perspectives of stakeholders when developing new products and services, and ii) to fill gaps in skills and expertise. Both require the ability to engage with - and form various types of relationships with - individuals and groups, drawing on a range of art skills and attributes such as communications and inter-personal skills; negotiation and influencing skills; and the intuitive ability to identify a suitable business partner.

Companies known for their successful record of innovation have often institutionalized collaboration within organizational systems and processes. For example, Buckley (2005; tinyurl.com/nykdaz3) report the implementation by Procter \& Gamble of a "connect and develop" program to promote open collaboration and idea-shar- 


\section{Strategic Innovation for Business Performance: The Art and Science of Transformation} Harold Schroeder

ing between employees in different areas of the organization and with external stakeholders. Similarly, Jaruzelski, Loehr, and Holman (2011; tinyurl.com/lysol6j) attribute the innovation success of the Visteon Corporation, a leading U.S.-based supplier of electronic products for automobiles, to the company's proactive efforts to formally increase collaboration between employees in different locations and with its joint venture partners. In many organizations, collaboration, jointworking, and knowledge-sharing are being facilitated by the use of Web 2.0 software (tinyurl.com/dqt86) such as "wikis" on company intranets (Bennett et al., 2010: tinyurl.com/mexxnk9; Fraser and Dutta, 2008: tinyurl.com/ m64vgsd; Bughin et al., 2010: tinyurl.com/lvo8etp).

The Internet and Web 2.0 technologies have revolutionized the ways in which businesses can now engage their customers in the innovation process, as well as providing a wealth of market intelligence about their preferences and characteristics. Companies that are making full use of their potential include Dell, which invites customers to submit their own suggestions for innovative products via the company's Direct2Dell blog (tinyurl.com/n8krna9), and Levi Strauss, which uses Facebook "likes" to generate information on consumer style preferences, thus providing a ready arena for the experimentation and commercialization stages of innovation.

The second common form of collaboration in the innovation process has evolved in response to the recognition that organizations often lack expertise in some stages of the innovation process, which prevents them from turning good ideas into value-generating products, services, or business models. In particular, innovative ideas generated by entrepreneurial firms are often stunted due to a lack of commercialization talent or funding. This problem has been considered particularly acute in Canada, where the Government has taken the initiative of establishing organizations such the Health Technology Exchange (htx.ca) and MaRS Innovation (marsinnovation.com) to promote collaboration and partnerships between entrepreneurs, venture capitalists, and others in Ontario's medical and assistive technologies sector, and to facilitate stakeholder input into the innovation process.

Major corporations that have traditionally been competitors also sometimes form partnerships in order to pool their expertise in developing new products and services. For example, SAP and RIM (now BlackBerry) reportedly worked collaboratively to provide access to SAP applications via the BlackBerry platform (IT On- line, 2008; tinyurl.com/n3yqww3). More commonly perhaps, firms may outsource their innovation processes to reduce risk and provide more favourable conditions for innovation (Chesbrough, 2003; tinyurl.com/aqkav9t), or they may acquire startups for the purpose of boosting innovation performance. This is a practice used by GE, for example (cited in De Souza et al., 2009; tinyurl.com/mzxbdj5). The trend of "open collaboration" involving sharing of ideas and joint experimentation between organizations within a network has been reported to contribute significantly to more rapid implementation, at lower cost, and reduced risk for the firms involved (Chesbrough and Appleyard, 2007: tinyurl.com/ 3ne6xts; Creamer and Amaria, 2012: tinyurl.com/m6ftjgd).

\section{Strategy}

It is clear from previous research that following a clearly-defined innovation strategy rather than an ad hoc approach is one of the preconditions for success in innovation. In a way, this seems counterintuitive: strategy implies constraints, and it might be argued that creativity should not be stifled in this way. But, most businesses cannot afford to waste time and resources in the development of ideas that are at a tangent to their organizational mission or core objectives. Having a strategy defines the broad scope within which innovations likely to deliver business value can be developed; as Favaro (2012; tinyurl.com/mqx8slf) points out, strategy is "the series of choices you make on where to play and how to win to maximize long-term value".

The innovation strategy should therefore be based on the organizational mission, core values, and business goals. It will define the goals and objectives of innovation and acceptable ways of meeting them (Anthony et al., 2008; tinyurl.com/l3fbhxl) and perhaps identify responsibilities for developing solutions within specified areas (De Souza et al., 2009; tinyurl.com/mzxbdj5). This strategy will not only help to ensure that value-generating innovations are developed, but also help motivate employees to come up with innovative solutions to specific organizational problems and issues (De Souza et al., 2009; tinyurl.com/mzxbdj5). The innovation goals can also be used to develop quantifiable metrics for the purpose of measuring innovation performance and reporting back to organizational leaders.

The science of innovation strategy development and implementation thus involves the systematic identification of problems or areas of interest, based on core business goals and techniques such as environmental 


\section{Strategic Innovation for Business Performance: The Art and Science of Transformation} Harold Schroeder

scans and market analyses. It also involves the ability to translate these problems into specific innovation objectives, goals, and metrics, while ensuring that targets and measurement activities do not stifle creativity and "out of the box" thinking. But, art is also fundamental to strategy formulation, especially in the context of innovation. As highlighted by the example of Steve Jobs at Apple Inc., understanding the market and emerging business opportunities requires not only the ability to understand facts and figures but it is also largely a personal, intuitive skill. Moreover, strategy deployment requires the ability to effectively communicate effectively with a range of stakeholders, including employees, suppliers, customers, and others, convincing them of the need for innovation and the importance of thinking and behaving in new ways to achieve the defined goals. This deployment involves understanding the perspectives and likely concerns of these stakeholder groups, translating the innovation strategy and goals into appropriate language, and developing incentives that act as drivers of change.

\section{Infrastructure}

Researchers are also increasingly flagging up the need to "institutionalize" (Anthony et al., 2008; tinyurl.com/ 13fbhxl) innovation by establishing organizational systems and structures to support various stages of the process. There is a common tendency to think of innovation in terms of the generation of new ideas for products and services, but this is only the starting point of innovation. Successful innovations are those that are actually implemented and deliver value to the organization and its customers and, as such, involve many stages of work and different functional areas. For example, De Souza and colleagues (2009; tinyurl.com/ mzxbdj5) identified five key stages in the innovation process: i) idea generation and mobilization; ii) screening and advocacy; iii) experimentation or prototype building; iv) commercialization; and v) diffusion and implementation.

Innovation therefore requires structures and systems to support each stage of the innovation process, helping to ensure that adequate resources and facilities are allocated to each phase of work, and that responsibilities and accountabilities are clearly defined. After an innovative idea has been generated, for example, it needs to be evaluated and prioritized within the overall innovation strategy and in relation to immediate and longerterm business needs. This process ideally involves screening by a dedicated innovation strategy team with oversight of the whole business, to ensure that the in- terests of individual areas do not bias the outcomes. Such a team can also act as an advocate of innovation when the organization is making decisions and allocating resources.

Many successful innovators also establish other new structures or organizations with responsibility for specific stages or aspects of innovation. Anthony, Johnson, and Sinfield (2008; tinyurl.com/l3fbhxl) cite the examples of incubators or independent working groups to launch or accelerate the development of innovative ideas and training units that provide managers and employees with the skills and knowledge needed to become more innovative. Some organizations have chosen to minimize the risk and disruption of innovation to the core business by establishing spin-off organizations for the sole purpose of innovation, or by completely outsourcing this function (De Souza et al., 2009; tinyurl.com/ mzxbdj5).

The innovation infrastructure includes the organizational systems that must be realigned to support an innovative culture. Especially important are those systems that shape the ways that employees think and behave at work, including recruitment and selection, training and development, performance management, and reward and recognition systems. Modifying these systems to promote innovation may involve the use of extrinsic rewards such as career-progression opportunities, salary increases, and other forms of recognition, as well as the more intrinsic rewards of interesting work and opportunities for self-achievement. Developing appropriate systems requires knowledge and expertise in human resources, but it also requires an astute ability to understand what motivates different groups of employees to be more innovative. For example, researchers may be attracted more by opportunities for interesting work, whereas sales and marketing specialists might be encouraged by external targets and associated rewards. There is evidence of both approaches being used by successful innovators: within IBM, the intrinsic reward of being associated with a prestigious organization has been used to attract managers to its emerging business organization, and Google offers employees stock options when their innovative ideas are successfully developed into new products (Philips, 2012; tinyurl.com/nykto5z).

\section{When Art and Science Are Ignored}

Though the above sections have highlighted good practice in innovation among well-known organizations, the literature also reveals many examples of less suc- 


\section{Strategic Innovation for Business Performance: The Art and Science of Transformation} Harold Schroeder

cessful attempts to innovate, even among major global firms. These examples can be linked in many cases to a lack of attention to the art and science of transformation.

For example, according to White and Farwell (2012; tinyurl.com/mnqstss), it has been the superior ability of Apple Inc. to establish a strong "culture of innovation", along with a more systematic approach to the innovation process, that has given it the edge over BlackBerry Ltd. (formerly Research In Motion Ltd.) in the smartphone market in recent years. The leadership skills of Steve Jobs, such as an ability to intuitively understand the market and anticipate future demands, are highlighted as having been major contributors to Apple's past record of successful innovation, with BlackBerry lacking such a strong leadership figure in its history. Moreover, despite some poor management practices in both firms, Apple successfully used its human-resources systems to encourage high performers to remain in the company and to ease weak performers out, while BlackBerry demonstrated no such ability to manage its performance through organizational systems in this way, and has shown a tendency to "grow like topsy" in a seemingly unplanned manner (White and Farwell, 2012; tinyurl.com/mnqstss).

Innovation efforts are also often hindered by a narrowsighted and risk-averse approach. This approach often occurs in larger, more successful companies that have invested heavily in the production of particular products and services and are almost exclusively focused on maximizing profits and increasing efficiencies within these same product lines rather than exploring new possibilities. The approach becomes ingrained in organizational cultures and operating practices and hinders innovation and flexibility even when market conditions change.

Kodak is an example of a company that experienced tremendous success in the film photography market, but has failed so far to adapt adequately to the digital photography market, in contrast to its more agile competitor Fujifilm. Contributing factors reportedly include a traditionally autocratic leadership style that has historically stifled innovation, and a failure to look holistically at the organization's whole business model and the need to adapt it to changing technologies (The Economist, 2012; tinyurl.com/7e5p6sf). It remains to be seen whether recent attempts to adopt a new business model focused on the delivery of products and services for managing digital image libraries - will be adequately supported by a transformation of company culture and operating systems (Hamm and Symonds, 2006: tinyurl .com/m2gm4k2; The Economist, 2012: tinyurl.com/7e5p6sf). Another example from the literature of a firm that failed to adopt a sufficiently holistic approach to innovation is the baby food producer Gerber Foods. As Wessell (2012; tinyurl.com/ogat6w3) explains, this company recently tried to break into the adult food market simply by repackaging some of its existing products, a strategy focused on maximizing existing efficiencies and reducing costs, but which lacked creativity and was poorly aligned with the demands of the target market, resulting in the failure of the rebranded product line.

\section{Conclusion and Recommendations}

For organizations wishing to improve their innovation performance, this article highlights the need to focus on strategy, systems, culture, and collaboration, and to recognize the inter-relationships between them. Thus, organizations need to apply both art and science in a holistic process of transformation. What needs to be done in practice will vary between organizations, depending on their existing state of innovation-readiness and the nature of their corporate culture and organizational systems. However, a number of general recommendations relating to transformation for improved innovation performance can be made:

1. Develop a formal innovation strategy that identifies priority areas linked to the organization's mission and business goals, and is aligned with its core purpose and values. If these elements have not been formally articulated, this gap should be addressed before the innovation strategy is developed in order to avoid wasting time and resources.

2. Allocate dedicated resources and formal responsibilities for each stage of the innovation process, and ensure that the necessary infrastructure, skills, and expertise are made available, either within the organization or through collaboration with external bodies.

3. Conduct a review of organizational culture, structure, and systems - using an approach based on both art and science - to identify ways in which these aspects are likely to promote or hinder innovation, and identify the changes necessary to establish an innovation-conducive organizational environment. Culture can be investigated using methods such as surveys, interviews, and focus groups to explore the attitudes of employees and managers. Human-resource man- 


\title{
Strategic Innovation for Business Performance: The Art and Science of Transformation
}

\author{
Harold Schroeder
}

agement systems in particular should be systematically examined to identify ways in which they are currently rewarding or penalizing innovative thinking and behaviour.

4. Implement a cultural transformation strategy that is targeted at both individual employees and organizational systems. Ways of thinking and behaving at work can be influenced over time by a process of communicating the desired new norms and involving employees in discussions about how to apply these norms to their own areas of work. Organizational systems should be modified as necessary to ensure these systems are aligned with the new norms, including recruitment of managers with appropriate attitudes and management styles, and ensuring that innovative approaches and achievements are acknowledged and rewarded in the performance-management system and the compensation system.

5. Establish systems and tools for the purpose of measure and monitoring innovation performance against the strategy, including detailed plans, performance metrics, and reporting methods such as balanced scorecards. These systems and tools will keep innovation in the minds of organizational executives, managers, and employees; ensure that achievements are acknowledged; and help highlight remaining innovation barriers.

Trends in open collaboration and new opportunities to engage stakeholders through social media are improving the prospects for successful innovation on the part of all organizations. Applying the "art and science of transformation" approach to organizational culture and systems can help ensure that the potential benefits of these developments can be secured.

\section{About the Author}

Harold Schroeder is President of Schroeder \& Schroeder Inc., a Toronto-based firm of senior program managers and project managers, management consultants, and corporate managers. By focusing on both the art and science of transformation, the firm assists organizations who are planning and implementing major transformation initiatives and who have had, or currently are experiencing, sub-optimal business results through their strategic or operational transformation projects. Having worked many years in politically challenging and complex environments with demanding timelines and deliverables, Mr. Schroeder is recognized by clients for his superior relationship-management, problemsolving, communication, and negotiation skills. He has led various management consulting practices in large consulting firms and has acted as a consultant and project manager on over 150 consulting engagements for clients throughout North America and Europe. Most recently, Mr. Schroeder has been involved in significant projects in the areas of innovation and entrepreneurship, including projects for both private sector clients and government ministries. He has also published and presented on the topic of innovation and entrepreneurship.

Citation: Schroeder, H. 2013. Strategic Innovation for Business Performance: The Art and Science of (cc) BY Transformation. Technology Innovation Management Review. September 2013: 6-12.

Keywords: innovation, transformation, art and science of transformation, strategy, organizational change 